\title{
Cruzamientos interpoblacionales en Mytilus chilensis, un bivalvo de importancia comercial y sus efectos sobre el crecimiento en longitud de la valva durante la etapa larval
}

\author{
Inter-population breeding in Mytilus chilensis, an economically important bivalve, \\ and its effects on the shell length during the larval stage \\ JE Toro ${ }^{a *}$, AC Alcapán ${ }^{\mathrm{a}}$, RA Stead ${ }^{\mathrm{b}}$ \\ anstituto de Biología Marina "Dr. Jürgen Winter", Universidad Austral de Chile, Valdivia, Chile. \\ bI-MAR y Depto. de Recursos Naturales y Medio Ambiente, Universidad de Los Lagos, Puerto Montt, Chile.
}

\begin{abstract}
SUMMARY
Two geographically separated natural populations of Mytilus chilensis were utilized to carry out the experimental crosses on the present study. In every crossing, using the factorial design with replication, fertilization of eggs occurred without detection of significant differences among inter and intra-population crosses in relation to percentage of eggs developed into larvae at day $4(\mathrm{P}>0.05)$. However, the percentage of abnormal larvae at day 4 , was significantly higher among inter-population crosses $(\mathrm{P}<0.05)$. The larvae from each cross were placed into a 2001 fiber-glass tank containing 1 $\mu \mathrm{m}$ filtered and U.V. treated fresh sea water, at a density of 100 larvae per ml. A high cell concentration of the micro algae Isochrysis galbana was used as food. Samples for analyzing larval growth were taken from the larval cultures at 4, 10 and 20 days after fertilization. Larval samples were videotyped from a plankton decantation chamber in an inverted microscope fitted with a Pulnex video camera. Selected images were captured for subsequent processing and measurement of each larva using a Scion Image 3.0b PC Software. Significantly differences $(\mathrm{P}<0.05)$ were found in the size of the larvae among the experimental crosses. The sibs from inter-population crosses showed significantly $(\mathrm{P}<0.05)$ higher sizes than those produced by the intra-population crosses. These higher values in the shell length of inter-population juvenile mussels may suggest the presence of heterosis.
\end{abstract}

Palabras clave: heterosis, bivalvos, Mytilus, Chile.

Key words: inter population crosses, heterosis, bivalves, Mytilus, Chile.

\section{INTRODUCCION}

Los cultivos marinos han sido identificados como una alternativa de desarrollo económico y social de un amplio sector productivo nacional e internacional (Ottolenghi y col 2005). La zona costera del sur de Chile es muy apropiada para el desarrollo de la acuicultura de moluscos (Winter y col 1984, Navarro y Gutiérrez 1990, Barton 1997, Sánchez 2002, Alcapán y col 2007), debido a sus numerosos estuarios y bahías protegidas alejadas de fuentes de contaminación industrial. Entre las especies de moluscos importantes desde el punto de vista comercial para la acuicultura está el "chorito chileno" (Mytilus chilensis), recurso pesquero que incrementó su desembarque de $3.352 \mathrm{t}$ en 1992 a 89.797 t en el 2005 (SERNAPESCA 2006). Sin embargo, hasta el momento, el explosivo incremento de la acuicultura en Chile se debe principalmente a un aumento en el número de concesiones otorgadas y no a un aumento de la productividad de los cultivos (Barton 1997, Toro y col 2004a ${ }^{a}$, Alcapán y col 2007). El desarrollo a futuro de la acuicultura debería considerar todos los aspectos relacionados con la biología de las especies incluyendo

Aceptado: 09.04.2008.

* Instituto de Biología Marina, Universidad Austral de Chile, Casilla 567, Valdivia, Chile; jtoro@uach.cl el manejo genético de las mismas (Newkirk 1980, Toro y col 1995, Beaumont y Hoare 2003, Toro y col 2004a, Alcapán y col 2007).

Los moluscos bivalvos en general tienen un crecimiento lento y además presentan una alta variabilidad en el crecimiento (Winter y col 1984, Beaumont y Hoare 2003, Toro y col 2004 ${ }^{\mathrm{a}}$ ). Esto último significa que no todos los individuos de una cohorte alcanzan la talla comercial en el mismo tiempo, haciendo por ello más difícil la labor al momento de su comercialización. El desarrollo de "cepas" producidas a nivel comercial que tengan una mayor tasa de crecimiento y/o una menor variabilidad en el crecimiento incrementaría el atractivo económico para llevar a cabo su cultivo.

Existe en la literatura evidencia sobre una correlación positiva entre la tasa de crecimiento y la heterocigosidad multilocus (HML) (Hansson y Westerberg 2002) en poblaciones naturales de bivalvos marinos (Zouros y col 1980, Garton y col 1984, Koehn y Gaffney 1984, Toro y col 1996, Toro y Vergara 1999). La hibridación es una técnica comúnmente utilizada para el manejo genético y su éxito es por lo general atribuido al incremento de la heterocigosidad (Frankel 1983, Mitton y Grant 1984, Koment y col 2002, Beaumont y Hoare 2003). Estudios de hibridación intraespecífica, utilizando poblaciones aisladas geográficamente en Crassostrea virginica, indican que 
se logra obtener un incremento en la tasa de crecimiento y una mayor sobrevivencia en la progenie producto de hibridación (Mallet 1982, Mallet y Haley 1983). De esta forma, los efectos de heterosis producto de la hibridación pueden teóricamente ser obtenidos a través de cruzamientos entre poblaciones aisladas geográficamente o por cruzamientos de líneas endogámicas (Wilkins 1981, Frankel 1983, Beaumont y Hoare 2003). Mediante métodos de hibridación, es posible explotar la varianza genética no aditiva (Mallet 1982), lo cual permite la obtención de ganancias en caracteres métricos mediante la explotación del vigor híbrido o heterosis (Mallet y Haley 1983, Toro y Aguila 1995). La utilización de poblaciones de M. chilensis aisladas geográficamente, con el mayor grado de distancia genética (Toro y col $2004^{\text {b }}$ ) en cruzamientos interpoblacionales, permite evaluar la posible obtención de una mejora en el crecimiento, producto del vigor híbrido.

\section{MATERIAL Y METODOS}

El análisis genético-poblacional de Mytilus chilensis llevado a cabo por Toro y col $\left(2004^{\mathrm{b}}\right)$ determinó que las poblaciones que garantizaban su adaptación genética al ambiente local (Koehn 1991) eran las provenientes de la

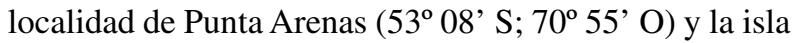
de Chiloé ( $41^{\circ} 51^{\prime} \mathrm{S} ; 7^{\circ} 50^{\prime} \mathrm{O}$ ). Se utilizó un total de 400 reproductores provenientes de cada población natural con el fin de realizar cruces tanto intra como interpoblacionales (híbridos), a través de un diseño factorial, conteniendo todos los cruzamientos dialélicos. Los cuatro desoves (4 réplicas), cada 4 días, se llevaron a cabo en forma individual recolectándose gametos de cada ejemplar en envases de 2 litros conteniendo agua filtrada y esterilizada con ultravioleta. Una vez obtenidos los gametos de cada ejemplar que desovó en cada una de las réplicas se procedió a mezclar las ovas y los espermios generando las familias para cada población y los híbridos con sus respectivos cruzamientos recíprocos. Un total de 6 familias (3 intrapoblacionales y 3 híbridas) se obtuvieron como mínimo en cada una de las réplicas. Los cultivos larvales se realizaron en estanques de 200 L, de acuerdo a la metodología descrita anteriormente por Toro y col (2003). Con el fin de no reducir la variabilidad genética, durante la etapa larval se utilizó una malla de plancton de entremalla adecuada para no producir selección por tamaño durante los cambios de agua de los estanques de cultivo larval. La densidad larval se mantuvo los primeros 10 días en 5 larvas/ml (DiSalvo y col 1984) y los 10 días siguientes en 1 larva/ml (Toro y col 2003). Existen trabajos en los cuales se describe que los primeros 20 días del cultivo larval en bivalvos es la etapa de mayor mortalidad (Mallet y col 1985, Helm y col 2006), alcanzando en la naturaleza valores por sobre el 85\% (Morgan 1995). El presente estudio se diseñó durante esta etapa del ciclo de vida de $M$. chilensis, dado que es durante este período donde las larvas nadan libremente y es posible controlar en mejor forma el número de indivi- duos por unidad de volumen, otorgándoles así las mismas condiciones ambientales y de alimentación. El recuento de larvas y mediciones de longitud se llevó a cabo a los 4, 10 y 20 días postfecundación, en base a muestras tomadas en cada estanque. La muestra de cada una de las familias se fijó en tubos Eppendorf con formalina al 10\%. Se midió la longitud máxima $(\mu \mathrm{m})$ de 120 individuos aproximadamente de cada una de las familias, utilizando un analizador de imágenes. Las larvas en portaobjetos fueron grabadas con una cámara de video Pulnex conectada a un microscopio invertido (Zeiss IM35), posteriormente las imágenes fueron capturadas utilizando un computador equipado con una tarjeta de video ATI. La longitud de las larvas se obtuvo usando el programa computacional Scion Image. Previo al análisis sobre el porcentaje de larvas anormales y sobrevivencia, los datos fueron transformados a arcoseno. El test no-paramétrico Kruskal-Wallis se utilizó para analizar el porcentaje de anormales y sobrevivencia, dado que estos datos mostraron una heterogeneidad de varianza significativa (test de Cochran). Del análisis de datos provenientes de los cruzamientos interpoblacionales, además de obtener información del crecimiento larval para cada cruzamiento y sus réplicas, se estimó el porcentaje de heterosis (Mallet 1982), utilizando la siguiente fórmula:

$$
\% \text { Heterosis }=\frac{\text { Heterosis }}{\mathrm{AB}} \times 100
$$

Donde: Heterosis $=$ Promedio del cruce interpoblacional $-\mathrm{AB} y$ $\mathrm{AB}=$ Promedio crecimiento de las poblaciones parentales.

\section{RESULTADOS Y DISCUSION}

Se observó fertilización en todos los cruzamientos y la división comenzó entre los 30 y 40 minutos a contar de la activación del huevo. No se detectaron diferencias significativas $(\mathrm{P}>0,05)$ entre los cruzamientos intra e interpoblacionales con respecto al porcentaje de ovas que se desarrollaron a larvas (cuadro 1). Sin embargo, el porcentaje de larvas anormales al día 4 y la proporción de larvas véliger que sufrieron mortalidad en los primeros estadíos de crecimiento fueron significativamente superiores $(\mathrm{P}<0,05)$ en los cruzamientos interpoblacionales (cuadro 1). Esta menor sobrevivencia podría estar reflejando leves diferencias en el tiempo de maduración o calidad de gametos entre los reproductores de ambas poblaciones (Toro y col 2002), ello a causa de las distintas condiciones de temperatura a que estuvieron sometidos previo al desove, ya que hay varios grados de latitud de diferencia, lo que conlleva a tener distintos períodos de desove.

Los valores de sobrevivencia progresivamente mayores obtenidos en los sucesivos desoves (réplicas) sugieren que una pobre condición de maduración gamética del reproductor puede afectar en forma adversa el éxito del cultivo 
larval, y enfatiza la necesidad de seleccionar reproductores en buena condición para llevar a cabo los cruzamientos en laboratorio (Chaparro y Winter 1983, Chaparro y Sanhueza 1986, Chaparro 1990). La mortalidad larval acumulada al día 20 en las réplicas 1,2 y 3 podría estar indicando la presencia de gametos inmaduros que generaron un mayor número de larvas anormales, particularmente debido a que ninguno de los cultivos larvales sufrió contaminación bacteriana o de protozoos. La evacuación de un gran número de gametos inmaduros en los ejemplares utilizados en las primeras réplicas es además corroborada por el mayor número de ejemplares que emitieron gametos maduros en los siguientes desoves $(15,17,17$ y 30 en las réplicas $1,2,3$ y 4, respectivamente). Se detectaron variaciones entre las réplicas con respecto al porcentaje de ovas desarrolladas, larvas anormales y sobrevivencia larval en ambos cruzamientos (intra e interpoblacionales), aunque los patrones observados dentro de cada réplica fueron consistentes (cuadro 1).
Los datos de longitud de la valva en larvas de 20 días, analizados por medio de un análisis de varianza anidado, confirmaron que no existían diferencias significativas entre los desoves (réplicas) dentro de cada grupo (cruzamiento) $(\mathrm{P}>0,05)$. En las larvas provenientes de cruzamientos interpoblacionales se observó un crecimiento significativamente mayor $(\mathrm{P}<0,05)$ que en aquellas provenientes de cruzamientos intrapoblacionales (cuadro 2), aunque sus valores están dentro de los rangos descritos para larvas de Mytilus edulis en experimentos realizados por Mallet y col (1985) y experimentos con diferentes regímenes alimentarios llevados a cabo por Bayne (1965).

Se observó además una diferencia significativa entre el crecimiento de las larvas provenientes de los cruzamientos interpoblacionales recíprocos, siendo las larvas producto del cruzamiento de la hembra de Punta Arenas con el macho de Chiloé las que presentaron un mayor crecimiento (cuadro 2).

Cuadro 1. Porcentaje de ovas con desarrollo a larva al día 4, porcentaje de de larvas anormales al día 4 y porcentaje de larvas véliger al día 20. $\mathrm{PA}=$ Punta Arenas, $\mathrm{Ch}=$ Chiloé, $\mathrm{PA} \times \mathrm{Ch}=$ hembra $\mathrm{PA} \times$ macho $\mathrm{Ch}, \mathrm{Ch} \times \mathrm{PA}=$ macho PA $\times$ hembra Ch. $\mathrm{H}=\mathrm{Test}$ estadístico de Kruskal Wallis entre los promedios de los cruzamientos intra e interpoblacionales. ns $=$ no significativo, $* *=P<0,01$.

Percentage of eggs which developed into larvae at day 4, percentage of abnormal larvae at day 4 and percentage of veliger larvae which survived to day 20. $\mathrm{PA}=$ Punta Arenas, $\mathrm{Ch}=$ Chiloé, $\mathrm{PA} \times \mathrm{Ch}=$ female PA $\times$ male $\mathrm{Ch}, \mathrm{Ch} \times \mathrm{PA}=$ male PA $\mathrm{x}$ females Ch. $\mathrm{H}$, Kruskal-Wallis statistic test between the mean performance of pure populations and that of inter-population crosses. $\mathrm{ns}=$ non significant, $* *=\mathrm{P}<0.01$.

\begin{tabular}{|c|c|c|c|c|c|c|c|c|c|c|c|c|}
\hline \multirow{2}{*}{ Réplica } & \multicolumn{4}{|c|}{ Porcentaje de desarrollados (día 4) } & \multicolumn{4}{|c|}{ Porcentaje de anormales (día 4) } & \multicolumn{4}{|c|}{ Porcentaje sobrevivencia (día 20) } \\
\hline & $\mathrm{PA}$ & $\mathrm{Ch}$ & $\mathrm{PA} \times \mathrm{Ch}$ & $\mathrm{Ch} \times \mathrm{PA}$ & $\mathrm{PA}$ & $\mathrm{Ch}$ & $\mathrm{PA} \times \mathrm{Ch}$ & Ch $\times$ PA & $\mathrm{PA}$ & $\mathrm{Ch}$ & $\mathrm{PA} \times \mathrm{Ch}$ & $\mathrm{Ch} \times \mathrm{PA}$ \\
\hline 1 & 69,1 & 61,4 & 60,5 & 57,3 & 30,8 & 25,1 & 62,1 & 59,1 & 26,3 & 28,1 & 11,3 & 5,1 \\
\hline 2 & 76,5 & 68,3 & 71,8 & 68,1 & 23,2 & 21,3 & 50,3 & 55,9 & 24,8 & 32,4 & 10,6 & 8,8 \\
\hline 3 & 89,6 & 76,9 & 84,9 & 72,6 & 24,4 & 15,7 & 44,2 & 48,8 & 49,2 & 44,7 & 18,6 & 10,9 \\
\hline 4 & 87,2 & 85,3 & 87,1 & 90,5 & 12,6 & 9,6 & 39,5 & 45,9 & 68,5 & 69,5 & 30,8 & 15,5 \\
\hline Prom & \multicolumn{2}{|c|}{$76,78 \pm 10,09$} & \multicolumn{2}{|c|}{$74,1 \pm 12,36$} & \multicolumn{2}{|c|}{$20,33 \pm 7,12$} & \multicolumn{2}{|c|}{$50,72 \pm 7,76$} & \multicolumn{2}{|c|}{$46,2 \pm 19,24$} & \multicolumn{2}{|c|}{$13,95 \pm 7,93$} \\
\hline $\mathrm{H}$ & \multicolumn{4}{|c|}{$0,370 \mathrm{~ns}$} & \multicolumn{4}{|c|}{$12,558 * *$} & \multicolumn{4}{|c|}{$15,122 * *$} \\
\hline
\end{tabular}

Cuadro 2. Promedio de la longitud valvar $(\mu \mathrm{m} \pm \mathrm{EE})$ de larvas véliger de Mytilus chilensis provenientes de los cruzamientros intra e interpoblacionales, luego de un período de crecimiento en laboratorio ( $20 \mathrm{~d})$. Resultados de una ANDEVA anidada y test de Tukey (para las abreviaciones ver tabla 1 ). $\mathrm{ns}=$ no significativo, $* * * \mathrm{P}<0,001$.

Mean shell length $(\mu \mathrm{m} \pm \mathrm{SE}$ ) of veliger larvae from Mytilus chilensis obtained by intra and inter-population crosses after a period of growth in the laboratory ( $20 \mathrm{~d}$ ). Results of a nested ANOVA and Tukey's HSD test (for abbreviations, see table 1). ns $=$ non significant, $* * * \mathrm{P}<0.001$.

\begin{tabular}{|c|c|c|c|c|}
\hline $\begin{array}{c}\mathrm{PA} \\
176,23 \pm 5,22 \\
\end{array}$ & $\begin{array}{c}\text { Ch } \\
169,79 \pm 4,8\end{array}$ & & $\begin{array}{c}\text { PA x Ch } \\
207,13 \pm 3,24\end{array}$ & $\begin{array}{c}\text { Ch x PA } \\
191,38 \pm 4,66 \\
\end{array}$ \\
\hline Factor & Fuente & $g l$ & $\mathrm{SC}$ & $\mathrm{F}$ \\
\hline \multirow[t]{3}{*}{ Longitud valva } & Cruzamiento & 3 & 17,422 & $101,273 * * *$ \\
\hline & Réplicas (cruzamiento) & 4 & 0,279 & $0,685 \mathrm{~ns}$ \\
\hline & Error & 1473 & & \\
\hline \multicolumn{5}{|c|}{ Test de Tukey } \\
\hline & \multicolumn{2}{|l|}{$\mathrm{PA}$} & $\mathrm{Ch}$ & $\mathrm{PA} \times \mathrm{Ch}$ \\
\hline $\mathrm{Ch}$ & \multicolumn{2}{|l|}{$\mathrm{ns}$} & - & - \\
\hline $\mathrm{PA} \times \mathrm{Ch}$ & \multicolumn{2}{|l|}{$* * *$} & $* * *$ & - \\
\hline $\mathrm{Ch} \times \mathrm{PA}$ & \multicolumn{2}{|l|}{$* * *$} & $* * *$ & $* * *$ \\
\hline
\end{tabular}


En base a los tamaños larvales significativamente mayores alcanzados en los cruzamientos interpoblacionales, se sugiere la presencia de vigor híbrido ya que el porcentaje de heterosis estimado para la talla valvar a los 20 días fue de 19,72\% para el cruzamiento entre hembras de Punta Arenas y machos de Chiloé y de 10,61\% para el cruzamiento recíproco. Estos valores de heterosis son comparables con los descritos por Mallet (1982) en Crassostrea virginica y los estimados en cruzamientos interpoblacionales de Ostrea chilensis por Toro y Aguila (1995). Los porcentajes de heterosis estimados podrían reflejar la presencia de efectos genéticos no aditivos para la variable longitud de la valva a los 20 días de edad en M. chilensis (Falconer 1981). Sin embargo, no están claras las ventajas que la heterosis estimada en la etapa larval podrían tener más adelante en la etapa juvenil y adulta. Resultados similares han sido descritos por Beaumont y col (1993) para cruzamientos entre Mytilus edulis y Mytilus galloprovincialis y también por Freeman y col (1994) para cruzamientos entre M. edulis y M. trossulus. El crecimiento en bivalvos parece estar relacionado con la conformación genética de los individuos (Newkirk 1980, 1983). La hibridación interpoblacional debería incrementar la heterocigosidad de los individuos (Argue y Dunham 1999, Lutz 2001, Beaumont y Hoare 2003), lo cual podría en parte explicar este mayor crecimiento observado en los cultivos larvales provenientes de cruzamientos interpoblacionales en el presente estudio. Sin embargo, esta mayor tasa de crecimiento en las larvas de cruzamientos interpoblacionales debe contraponerse con la baja sobrevivencia en la mayoría de los cruzamientos interpoblacionales, especialmente causada por diferencias en el grado de madurez gonadal al estar sometidas a distintas presiones ambientales (Toro y col 2002), por ejemplo, diferencias en la temperatura del agua. Estas diferencias en el grado de madurez gonadal podrían evitarse en el futuro a través del acondicionamiento de los reproductores en el laboratorio, previo a los desoves (Sarkis y Lovatelli 2007).

Los resultados de este estudio aportan evidencia de que el cruzamiento interpoblacional de Mytilus chilensis produjo a nivel de cultivo larval ejemplares con una mayor tasa de crecimiento, lo que a su vez sugiere que las larvas tipo véliger realizarán su metamorfosis antes que aquellas provenientes de cruzamientos intrapoblacionales. Se requiere realizar a futuro investigaciones que incluyan el estudio del comportamiento de familias de poblaciones puras y de sus cruzamientos recíprocos, permitiendo de esta forma estimar el fitness relativo de cada familia sometida a distintas condiciones ambientales.

\section{RESUMEN}

Dos poblaciones naturales de Mytilus chilensis aisladas geográficamente fueron utilizadas para realizar los cruzamientos experimentales en el presente trabajo. En todos los cruzamientos, utilizando un diseño factorial con réplicas, ocurrió fertilización de las ovas, no detectándose diferencias significativas entre los cruzamientos intra e interpoblacionales en cuanto al porcentaje de ovas que desarrollaron larvas al día $4(\mathrm{P}>0,05)$. Sin embargo, el porcentaje de larvas anormales al día 4 fue significativamente mayor en los cruzamientos interpoblacionales $(\mathrm{P}<0,05)$. Las larvas de cada cruzamiento se cultivaron en estanques de fibra de vidrio de $200 \mathrm{~L}$, con agua de mar filtrada y esterilizada con ultravioleta, en una concentración de 100 larvas por mL. Una alta concentración de la microalga Isochrisis galbana se utilizó como alimento. Muestras para analizar el crecimiento larval se tomaron a $\operatorname{los} 4,10$ y 20 días posterior a la fecundación. Las muestras de larvas se filmaron con una cámara se sedimentación montada en un microscopio invertido, equipado con una cámara de video Pulnex. Las imágenes se capturaron a un computador en donde se procesaron para medir la longitud de las larvas a través de un procesador de imágenes (Scion Image 3.0b PC Software). Se encontraron diferencias significativas para la longitud de la larva entre los cruzamientos $(\mathrm{P}<0,05)$. Los juveniles provenientes de los cruzamientos interpoblacionales tuvieron tamaños significativamente mayores que aquellos producidos por los cruzamientos intrapoblacionales. Estos mayores valores en la longitud de la valva de los mitílidos juveniles provenientes de los cruzamientos interpoblacionales sugieren la presencia de heterosis.

\section{AGRADECIMIENTOS}

Este estudio es parte de los objetivos del proyecto FONDECYT 1050341.

\section{REFERENCIAS}

Alcapán AC, RF Néspolo, JE Toro. 2007. Heritability of body size in the Chilean blue mussel (Mytilus chilensis Hupé 1854): effects of environment and aging. Aquac Res 38, 313-320.

Argue BJ, RA Dunham. 1999. Hybrid fertility, introgression, and backcrossing in fish. Rev Fish Sci 7, 137-195.

Barton JR. 1997. Environment, sustainability and regulation in commercial aquaculture: The case of Chilean salmonid production. Geoforum $28,313-328$.

Bayne BL. 1965. Growth and the delay of metamorphosis of the larvae of Mytilus edulis (L.). Ophelia 2, 1-47.

Beaumont AR, MD Budd. 1983. Effects of self-fertilization and other factors on the early development of the scallop, Pecten maximus. Mar Biol 76, 285-289.

Beaumont AR, KS Kelly. 1989. Production and growth of triploid Mytilus edulis larvae. J Exp Mar Biol Ecol 132, 69-84.

Beaumont AR, AKM Abdul-Martin, R Seed. 1993. Early development, survival and growth in pure and hybrid larvae of Mytilus edulis and Mytilus galloprovincialis. J Mollus Stud 59, 120-123.

Beaumont AR, K Hoare. 2003. Biotechnology and Genetics in Fisheries and Aquaculture. Blackwell Science, Oxford, UK.

Chaparro OR, JE Winter. 1983. The effect of winter period, gametogenesis and spawning on the calorific content of soft parts in Mytilus chilensis. Aquaculture 32, 419-422.

Chaparro OR, MA Sanhueza. 1986. Desarrollo embrionario y larval del choro zapato Choromytilus chorus (Bivalvia, Mytilidae). Biol Pesq 15, 75-79.

Chaparro OR. 1990. Effect of temperature and feeding on conditioning of Ostrea chilensis Philippi 1845 reproductors. Aquac Fish Manag 21, 499-405.

Frankel R. 1983. Heterosis: Reappraisal of Theory and Practice. Springer Verlag, Berlin, Germany, Pp 1-290.

Freeman KR, KL Perry, TG DiBacco, DJ Scarratt. 1994. Observations on two mytilid species from a Nova Scotia mussel farm. Can Tech Rep Fish Aquat Sci 1969, 1-47.

Garton DW, RK Koehn, TM Scott. 1984. Multiple-locus heterozygosity and physiological energetics of growth in the coot clam, Mulinia lateralis, from a natural population. Genetics 108, 445-455.

Hansson B, L Westerberg. 2002. On the correlation between heterozygosity and fitness in natural populations. Mol Ecol 11, 2467-2474. 
Helm MM, N Bourne, A Lovatelli. 2006. Cultivo de bivalvos en criadero. Un manual práctico. FAO, Documento Técnico de Pesca $\mathrm{N}^{\circ} 471$, Italia.

Koehn RK, PM Gaffney. 1984. Genetic heterozygosity and growth rate in Mytilus edulis. Mar Biol 82, 1-7.

Koehn RK 1991. The genetics and taxonomy of species in the genus Mytilus. Aquaculture 94, 125-145.

Koment H, P Haffray, S Kaushik, M New, I Olsen, AE Linamo. 2002. Defining breeding goals for future sustainable aquaculture. World Aquaculture 33, 11-14.

Lutz CG. 2001. Practical Genetics for Aquaculture. Blackwell Science, Oxford, UK.

Mallet AL. 1982. Quantitative genetics of the Atlantic Canadian oyster, Crassostrea virginica (Gmelin). PhD Thesis, Dalhousie University, Halifax, Nova Scotia, Canada.

Mallet AL, LE Haley. 1983. Growth rate and survival in pure population matings and crosses of the oyster Crassostrea virginica. Can J Fish Aquat Sci 40, 948-954.

Mallet AL, E Zouros, KE Gartney-Kepkay, KR Freeman, LM Dickie. 1985. Larval viability and heterozygote deficiency in populations of marine bivalves: evidence from pair mating of mussels. Mar Biol 87, 165-172.

Mitton JB, MC Grant. 1984. Associations among protein heterozygosity, growth rate and developmental homeostasis. Annu Rev Syst Ecol 15, 479-499.

Morgan SG. 1995. Life and death in the plancton: larval mortality and adaptation. In: McEduard L (ed). Ecology of Marine Invertebrate Larvae. CRC Press LLC, Florida, USA, Pp 279-321.

Navarro JM, A Gutiérrez. 1990. Cultivo de moluscos en Chile: sinopsis sobre el estado actual del cultivo de mitílidos en Chile. En: Hernández A (ed). Cultivo de moluscos en América Latina. CIID-Canadá, Bogotá DE, Colombia, Pp 189-210.

Newkirk GF. 1980. Review of the genetics and the potential for selective breeding of commercially important bivalves. Aquaculture 19, 209-228.

Newkirk GF. 1983. Applied breeding of commercially important molluscs: a summary of discussion. Aquaculture 33, 415-422.

Ottolenghi F, C Silvestri, P Giordano, A Lovatelli, MB New. 2005. Capture-based aquaculture. FAO, Rome, Italy.

Sánchez V. 2002. Crece la industria mitilicultora. Aquanoticias 75, 8-14.
Sarkis S, A Lovatelli. 2007. Instalation and operation of a modular bivalve hatchery. FAO Fisheries Technical Paper No 492, Roma, Italia.

SERNAPESCA, Servicio Nacional de Pesca. Anuario estadístico de Pesca, 2006. Ministerio de Economía, Fomento y Reconstrucción, Chile, Pp 291.

Toro JE, PR Aguila. 1995. Cruzamientos factoriales con cinco poblaciones de ostra chilena (Ostrea chilensis Philippi 1845) aisladas geográficamente. Arch Med Vet 27, 69-80.

Toro JE, MA Sanhueza, JE Winter, P Aguila, AM Vergara. 1995. Selection response and heritability estimates for growth in the Chilean oyster (Ostrea chilensis Philippi 1845). J Shellfish Res 14, 87-92.

Toro JE, AM Vergara, R Galleguillos. 1996. Multiple-locus heterozygosity, physiology and growth at two different stages in the life cycle of the Chilean oyster Ostrea chilensis. Mar Ecol P S 34, 151-158.

Toro JE, AM Vergara. 1999. Growth and heterozygosity in a 12-monthold cohort of Ostrea chilensis (Philippi, 1845) obtained by mass spawning in the laboratory. Mar Ecol PSZN 19, 311-323.

Toro JE, RJ Thompson, DJ Innes. 2002. Reproductive isolation and reproductive output in two coexisting mussel species (Mytilus edulis, M. trossulus) and their hybrids from Newfoundland. Mar Biol 141, 897-909.

Toro JE, AC Alcapán, AM Vergara, JA Ojeda. 2003. Heritability estimates of larval and spat shell height in the Chilean blue mussel (Mytilus chilensis Hupe 1854) produced under controlled laboratory conditions. Aquac Res 35, 56-61.

Toro JE, AC Alcapán, JA Ojeda, AM Vergara. 2004ª . Selection response for growth rate (Shell height and live weight) in the Chilean blue mussel (Mytilus chilensis Hupe 1854). J Shellfish Res 23, 753-757.

Toro JE, JA Ojeda, AM Vergara. 2004 ${ }^{\mathrm{b}}$. The genetic structure of Mytilus chilensis (Hupe 1854) populations along the Chilean coast based on RAPDs analysis. Aquac Res 35, 1466-1471.

Wilkins NP. 1981. The rationale and relevance of genetics in aquaculture: an overview. Aquaculture 22, 209-228.

Winter JE, JE Toro, JM Navarro, GS Valenzuela, OR Chaparro. 1984. Recent developments, status and prospects of moluscan aquaculture on the Pacific coast of South America. Aquaculture 39, 95-134.

Zouros E, SM Singh, HE Miles. 1980. Growth rate in oysters: an overdominant phenotype and its possible explanations. Evolution 34, 856-867. 
